\title{
ESTIMAÇÃO DE ESTADOS HARMÔNICOS USANDO ESTRATÉGIAS EVOLUTIVAS
}

\author{
E. F. Arruda, Student Member, IEEE* \\ elcio.arruda@ieee.org
}

\author{
N. Kagan, Senior Member, IEEE* \\ nelsonk@pea.usp.br
}

${ }^{*}$ ENERQ - USP

\section{RESUMO}

O objetivo deste trabalho é apresentar uma nova metodologia para estimar a distorção harmônica em um sistema elétrico, a partir de medições em locais específicos. O algoritmo desenvolvido se baseia em Estratégias Evolutivas. Esta técnica compreende um ramo de desenvolvimento dos Algoritmos Evolutivos, no qual se inserem os Algoritmos Genéticos. As vantagens no uso desta técnica residem nas facilidades de modelagem e em encontrar uma solução para problemas complexos. A maioria das técnicas encontradas na literatura para solução deste problema utiliza sincronização das medições por meio de métodos de alta tecnologia como GPS. O algoritmo proposto visa sincronizar os dados de forma offline por meio das informações do fluxo de carga para a freqüência fundamental, o que o torna muito mais econômico para compor um sistema de monitoramento de qualidade de energia. $\mathrm{O}$ algoritmo foi aplicado em uma rede de teste cujos dados foram extraídos do IEEE.

PALAVRAS-CHAVE: Estimação harmônica, distorções harmônicas, algoritmos evolutivos, estratégias evolutivas, qualidade de energia.

\section{ABSTRACT}

This paper aims at presenting a new methodology in order to estimate harmonic distortions in a power system, based on measurements in some given sites. The algorithm utilizes Evolutionary Strategies. This technique is a development

Artigo submetido em 24/03/2008 (Id.: 00864)

Revisado em 22/08/2008, 13/12/2008

Aceito sob recomendação do Editor Associado Prof. Julio Cesar Stacchini Souza branch of evolutionary algorithms within which genetic algorithms are included. The main advantage in using such technique relies upon its modeling facilities as well as its potential to solve fairly complex problems. Most papers available in the Scientific Literature base the problem by solving the algorithm on synchronized measurements, by means of high technology GPS power quality meters. The proposed method exposed in this paper synchronizes harmonic distortion data from various power quality meters in an offline way through a previous base case fundamental frequency load flow, what makes the power quality monitoring system much less costly. The algorithm is applied to an IEEE test case network.

KEYWORDS: Harmonic estimation, harmonic distortion, evolutionary algorithms, evolutionary strategies, power quality.

\section{INTRODUÇÃO}

Mensurar o impacto do conteúdo de fontes harmônicas no desempenho de um sistema elétrico consiste num aspecto importante em Qualidade de Energia.

Supondo conhecidos os locais e o conteúdo harmônico injetado no sistema elétrico, medidas que mitiguem o impacto das distorções em outras barras do sistema podem ser projetadas com a utilização de filtros passivos ou ativos. Todavia, na maioria das vezes, as fontes de distorções não são conhecidas (Du et al., 1999).

Embora o custo dos medidores de qualidade de energia tenha atingido valores cada vez mais baixos, ainda é inviável a utilização de medidores em todas barras de um sistema elétrico real, pois o monitoramento se tornaria demasiadamente 
oneroso.

Sendo assim, torna-se necessária a utilização de um algoritmo capaz de estimar, a partir de um pequeno número de pontos de medição, os valores das distorções harmônicas nos demais pontos do sistema. Desta forma, o algoritmo de Estimação de Estado Harmônico (EEH) compreende o processo reverso dos processos de simulação. Os simuladores analisam a resposta de um sistema elétrico dada a injeção de corrente harmônica em um ou mais pontos do sistema, enquanto os estimadores indicam os valores de injeção harmônica a partir das respostas do sistema elétrico por meio de medições (Heydt, 1989).

A metodologia de EEH consiste em uma ferramenta eficiente e econômica para o monitoramento do conteúdo harmônico em um sistema elétrico de potência. Um estimador harmônico pode ser formulado a partir da topologia da rede elétrica, das matrizes de admitâncias para as frequiências harmônicas e da localização de medidores (Du et al., 1998).

A utilização de algoritmos da área de Sistemas Inteligentes, como Estratégia Evolutiva (EE) proposta neste artigo, tornase viável por sua facilidade de implementação e sua velocidade em encontrar uma solução dentro do espaço de soluções frente às técnicas tradicionais.

Estimar o estado de uma rede quanto ao nível de distorção harmônica é um problema naturalmente complexo, por exigir uma confiança mínima nas informações provenientes de medidores de qualidade de energia. Além da segurança quanto à calibração do medidor, ao método de transmissão dos dados, à fidelidade da rede utilizada nas simulações frente à rede real, entre outros fatores capazes de acarretar discrepâncias entre o sistema real e o sistema simulado, há o problema da sincronização das informações provenientes dos medidores.

Tratando dos métodos de EEH propriamente ditos, há diversas abordagens na literatura. Entre as referências encontradas sobre o assunto, Heydt (1989) apresenta um método para identificar fontes de sinais harmônicos no Sistema Elétrico de Potência (SEP). Já Meliopoulos et al. (1992) ilustra a importância da especificação das medições, bem como dos equipamentos utilizados no processo de estimação e Meliopoulos (1994) apresenta um método de EEH em que um GPS (Global Positioning System) foi utilizado para a sincronização dos dados de medição.

Este artigo propõe utilizar as formas de onda armazenadas pelos medidores e as informações provenientes do fluxo de carga (em regime permanente senoidal na freqüência de 60 $\mathrm{Hz}$ - industrial ou fundamental), para a sincronização dos dados de medição. Tal adoção se torna bastante viável por diminuir os custos de um sistema de EEH.
O item 2 deste artigo apresenta a formulação do problema de EEH, consistindo na determinação do estado da rede na freqüência fundamental, no ajuste dos fasores de componentes harmônicas de tensão e corrente para sincronização das medições e numa formulação matemática do modelo de EEH. O item 3 apresenta a alternativa de solução do problema por estratégias evolutivas, em que é apresentada a codificação dos indivíduos que representam possíveis soluções ao problema, os operadores evolutivos de mutação, recombinação e seleção, bem como a função de avaliação adotada. O item 4 descreve a análise de sensibilidade sobre os principais parâmetros da estratégia evolutiva, de forma a avaliar a influência de cada parâmetro na evolução do algoritmo. O item 5 trata de aplicação do algoritmo proposto, de forma a obter estimações de distorção harmônica em diferentes condições de instalação dos medidores. Destacam-se, ainda, as evoluções da função de avaliação ao longo das gerações. O item 6 apresenta as principais conclusões deste artigo.

\section{FORMULAÇÃO DO PROBLEMA DE ES- TIMAÇÃO DE ESTADO HARMÔNICO}

O problema de estimação de estado harmônico (EEH) consiste, conforme apontado anteriormente, na avaliação para cada ordem harmônica de interesse, do estado da rede naquela freqüência harmônica, a partir de medições de tensões (e correntes) em alguns locais da rede. $\mathrm{O}$ estado da rede é definido pelos valores de tensões obtidas em todas as barras da rede. Os locais da rede com medição de valores de tensão (e corrente) são normalmente disponibilizados por meio de um sistema de monitoramento de qualidade de energia. Como os medidores utilizados são de alto custo, é comum que sejam disponibilizadas medições em poucos locais.

Quando o algoritmo em questão é utilizado para todas as harmônicas relevantes na rede, a distorção harmônica total pode então ser estimada nas barras do sistema. O diagrama da Fig. 1 ilustra a metodologia proposta. Os blocos principais desta figura serão pormenorizados nos itens subseqüentes.

\subsection{Estado da rede na freqüência funda- mental}

O estado da rede na frequiência fundamental, representado pelo bloco (i) do diagrama da Fig. 1, pode ser obtido a partir de duas formas:

- fluxo de potência a partir do conhecimento das medições de potências ativa e reativa de cargas e do estado dos geradores;

- estimador de estado convencional, no qual são conheci- 


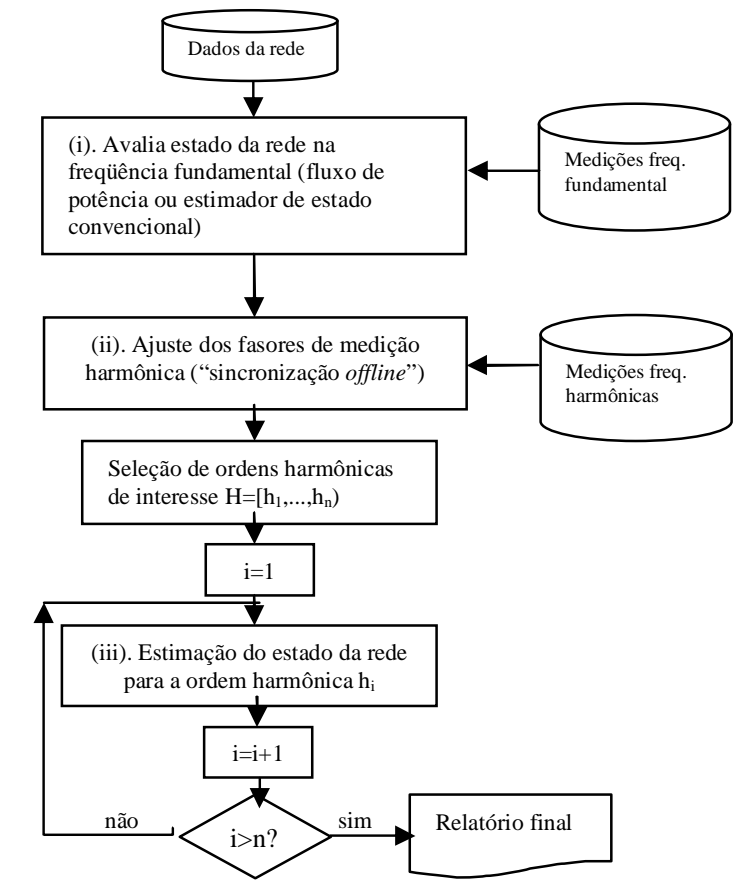

Figura 1: Fluxograma do método de estimação proposto

das as medições de potência e tensão, em componentes da rede (barras e ligações), compondo um conjunto de medições redundantes (em número superior ao de variáveis de estado da rede).

Neste artigo, o estado da rede para a freqüência fundamental é obtido por meio do fluxo de potência e métodos convencionais de cálculo. Assumem-se conhecidas as potências ativa e reativa nas barras de carga e as tensões nas barras de geração. Foi empregado o método de Gauss matricial, que se baseia na seguinte equação:

$$
\left[\mathbf{I}_{\mathbf{1}}\right]=\left[\begin{array}{l}
\mathbf{I}_{\mathbf{1} S} \\
\mathbf{I}_{\mathbf{1} C}
\end{array}\right]=\left[\mathbf{Y}_{\mathbf{1}}\right]\left[\mathbf{V}_{\mathbf{1}}\right]=\left[\begin{array}{cc}
\mathbf{Y}_{1}^{S S} & \mathbf{Y}_{1}^{S C} \\
\mathbf{Y}_{1}^{C S} & \mathbf{Y}_{1}^{C C}
\end{array}\right]\left[\begin{array}{l}
\mathbf{V}_{\mathbf{1} S} \\
\mathbf{V}_{\mathbf{1} C}
\end{array}\right]
$$

onde:

$\left[\mathbf{I}_{1}\right],\left[\mathbf{I}_{1 S}\right],\left[\mathbf{I}_{1 C}\right]$ representam as correntes injetadas em todas as barras, nas barras de suprimento e nas barras de carga, respectivamente.

$\left[\mathbf{V}_{1}\right],\left[\mathbf{V}_{1 S}\right],\left[\mathbf{V}_{1 C}\right]$ representam as tensões nodais em todas as barras, nas barras de suprimento e nas barras de carga, respectivamente.

[Y $\left.\mathbf{Y}_{1}\right]$ é a matriz de admitâncias nodais para a freqüência fun- damental, particionada de acordo com as barras de suprimento e as barras de carga.

Resulta que o vetor de tensões nas barras pode ser obtido a partir das tensões conhecidas nas barras de suprimento e das correntes conhecidas nas barras de carga:

$$
\left[\mathbf{V}_{\mathbf{1} C}\right]=\left[\mathbf{Y}_{1}^{C C}\right]^{-1}\left\{\left[\mathbf{I}_{\mathbf{1} C}\right]-\left[\mathbf{Y}_{1}^{C S}\right] \cdot\left[\mathbf{V}_{\mathbf{1} S}\right]\right\}
$$

Caso as correntes $\left[\mathrm{I}_{1 C}\right]$ sejam funções das tensões $\left[\mathrm{V}_{1 C}\right]$, como são os modelos de carga conhecidos (por exemplo, potência ou impedância constante), a equação (2) poderá ser resolvida iterativamente.

\subsection{Ajuste dos fasores de grandezas em cada ordem harmônica}

As medições de valores de grandezas, tensão ou corrente, em determinada ordem harmônica, num dado local da rede, são fornecidas em módulo e ângulo. Como os medidores dispõem também das grandezas medidas na freqüência fundamental, os ângulos das grandezas de determinada ordem harmônica podem ser referenciados às grandezas na freqüência fundamental.

A partir das defasagens na tensão fundamental entre as várias barras da rede, obtidas conforme explicado no item 2.1 (fluxo de potência), podem ser então sincronizados os ângulos dos fasores de todas as barras da rede em dada ordem harmônica, o que é representado pelo bloco (ii) da Fig. 1.

O fasor $\dot{V}_{1}^{k}$, referente à barra $k$ de um sistema elétrico com $n_{b}$ barras, resultante da solução do fluxo de potência na freqüência fundamental, pode ser escrito como:

$$
\dot{V}_{1}^{k}=V_{1}^{k} e^{j \theta_{1}^{k}}
$$

O algoritmo utiliza o estado da rede na freqüência fundamental, em particular os ângulos $\theta_{1}^{k}, k=1, \ldots, n_{b}$, para ajustar os ângulos dos fasores medidos nas diversas freqüências harmônicas. Desta forma, uma dada medição de tensão harmônica $h$ na barra $k$ genérica:

$$
\dot{V}_{h M}^{k}=V_{h M}^{k} e^{j \phi_{h M}^{k}}
$$

é ajustada para:

$$
\dot{V}_{h M}^{k}=V_{h M}^{k} e^{j\left(\phi_{h M}^{k}+\theta_{1}^{k}\right)}=V_{h M}^{k} e^{j \theta_{h M}^{k}}
$$

Este procedimento promove a sincronização dos dados de medição, de acordo com as variações em relação ao ângulo da tensão fundamental em cada barra $k$. 


\subsection{Estimação de estado para cada or- dem harmônica}

Conforme Fig. 1, a seleção das ordens harmônicas de interesse no estudo é submetida a um algoritmo de estimação do estado da rede, EEH, correspondente ao bloco (iii) do diagrama. Esta seleção ou escolha pode ser realizada em função das ordens harmônicas mais significativas para estudo, ou todas as harmônicas existentes, provenientes dos dados de medidores ou mesmo selecionadas pelo analisador. As ordens harmônicas de interesse compõem um vetor $\mathbf{H}$, que é dado por $\left[h_{1} \ldots h_{n}\right]^{t}$. O algoritmo de EE é então acionado para cada ordem harmônica do vetor $\mathbf{H}$.

Assume-se que sejam monitoradas as tensões harmônicas em determinadas barras da rede e que as distorções harmônicas sejam originadas pelas injeções de correntes harmônicas em determinados consumidores da rede.

Desta forma, o problema que se coloca é o de determinar, para uma dada ordem harmônica, quais são as injeções de correntes harmônicas nas barras da rede. Uma vez avaliadas as injeções (mais prováveis) de correntes harmônicas, podem ser avaliadas as tensões harmônicas em quaisquer barras da rede. O problema de EEH pode ser formulado conforme a seguir:

Determinar as correntes harmônicas injetadas nas $\left(n_{c}\right)$ barras de carga, $\dot{I}_{h}^{j}=I_{h}^{j} e^{j \delta_{h}^{j}}, j=1, \ldots, n_{c}$, de forma a minimizar a soma do erro quadrático entre valores medidos e calculados das tensões, dada por:

$$
\min \sum_{k=1}^{n_{m e d}}\left|e e_{h}^{k}\right|^{2}=\sum_{k=1}^{n_{m e d}}\left|\dot{V}_{h M}^{k}-\dot{V}_{h \text { Calc }}^{k}\right|^{2}
$$

sendo que as tensões calculadas nos medidores $\left(k=1, \ldots ., n_{m e d}\right)$ são avaliadas por:

$$
\dot{V}_{h \text { Calc }}^{k}=\sum_{j=1}^{n_{c}} \bar{Z}_{h}^{k j} \cdot \dot{I}_{h}^{j}
$$

onde:

$e e_{h}^{k}$ : é o erro de estimação na barra $k$, ordem harmônica $h$;

$\dot{V}_{h C a l c}^{k}$ : é a tensão calculada para uma determinada barra $k$, a partir de um indivíduo que representa as correntes injetadas nas barras para a ordem harmônica em análise;

$\dot{V}_{h M}^{k}$ : é a tensão harmônica medida na barra $k$;

$Z_{h}^{k j}$ : é o elemento $(k j)$ da matriz de impedâncias nodais na freqüência $h$, dada pela inversa da matriz de admitâncias: $\left[\mathbf{Z}_{\mathbf{h}}\right]=\left[\mathbf{Y}_{\mathbf{h}}\right]^{-1}$
O problema em questão pode ser resolvido por inúmeros algoritmos de busca heurística. Num algoritmo de busca exaustiva, por exemplo, deveriam ser variados os valores de correntes harmônicas injetadas nas barras (módulo e fase) e ser avaliado o erro médio quadrático para cada combinação. Outra técnica utilizada para a estimação emprega o método estatístico de Monte Carlo, em que são simuladas, aleatoriamente, um grande número de possíveis soluções (valores de correntes injetadas), para então, utilizando um critério de avaliação adequado ao problema, optar pela(s) melhor(es) solução(ões) que atenda(m) o critério de avaliação.

Este artigo, no entanto, utiliza estratégias evolutivas a fim de avaliar o conjunto de injeções de correntes que promove a mínima somatória dos erros quadráticos, conforme será contemplado no item 3. Cabe salientar que, após determinadas as injeções de correntes harmônicas, é possível estimar o estado da rede em qualquer barra da rede utilizando a equação (7), obtendo, desta forma, o estado da rede em qualquer ordem harmônica.

O valor de distorção harmônica total, para uma determinada barra $k$, pode ser definido após avaliadas as grandezas para todas as ordens harmônicas analisadas, conforme equação (8).

$$
D H T_{k}=\frac{\sqrt{\left(\sum_{i=2}^{\infty} V_{i}^{k^{2}}\right)}}{V_{1}^{k}}
$$

\section{ESTRATÉGIAS EVOLUTIVAS APLICA- DAS AO PROBLEMA DE ESTIMAÇÃO HARMÔNICA}

\subsection{Considerações Iniciais}

As Estratégias Evolutivas foram desenvolvidas por Rechenberg e Schwefel (Back and Schwefel, 1996; Back et al., 1997), que iniciaram os estudos neste campo nos anos 60 na Technical University of Berlin, na Alemanha.

Esta ferramenta emprega conceitos de evolução aplicados a uma população de indivíduos que representam, cada um, uma possível solução para o problema em estudo. Os processos ou operadores de mutação e recombinação não dependem, a priori, da natureza do problema. Já a formulação do indivíduo e o método de avaliação devem ser adaptados ao problema específico.

De forma geral, um algoritmo de EE pode ser descrito como segue: 


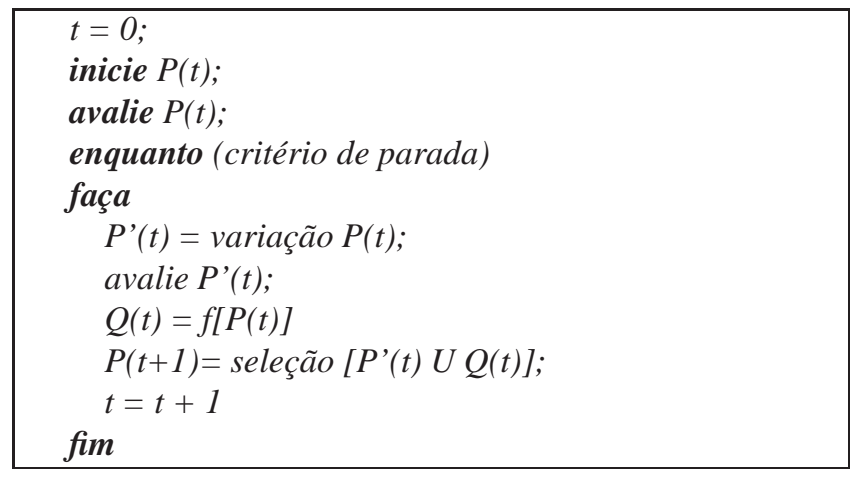

Neste algoritmo, $P(t)$ denota uma população de $\mu$ indivíduos na geração $t$, cuja inicialização pode ser realizada de forma aleatória (sorteio dos $\mu$ indivíduos). Cada indivíduo representa uma possível solução para o problema em análise, com representação estabelecida de acordo com uma codificação, conforme explicado no item 3.2. $Q(t)$ representa um conjunto de indivíduos que podem ser considerados para a seleção, conforme a função $f[]$. Por exemplo, $Q$ pode ser igual ao conjunto $P(t)$; no entanto, $Q$ também pode ser igual ao conjunto vazio. Uma nova geração de indivíduos $P^{\prime}(t)$ de tamanho $\lambda$ é gerada pela variação do conjunto $P(t)$ por meio dos operadores de recombinação e mutação, conforme exposto nos itens 3.3 e 3.4, respectivamente. Os novos indivíduos $P^{\prime}(t)$ são então avaliados medindo a "distância" entre cada um destes e a solução "ótima" do problema considerado. Como produto da avaliação, a cada indivíduo é atribuída uma nota (medida de adaptação, conforme item 3.5). Então, uma nova população é formada na iteração $t+1$, pela seleção dos indivíduos mais adaptados, conforme item 3.6.

Após determinado número de gerações, a condição de parada deve ser atendida, a qual usualmente indica a existência de um indivíduo na população que represente uma solução aceitável para o problema, ou quando o número máximo de gerações foi atingido (Back and Schwefel, 1996; Back et al., 1997).

Serão definidos nos itens seguintes, de forma mais pormenorizada, os operadores e formulações necessários para a utilização da estratégia evolutiva ao problema de EEH.

\subsection{Representação dos Indivíduos em EEH}

O indivíduo utilizado para a solução do problema em estudo deve representar um possível estado harmônico do sistema. Sendo assim, o indivíduo considerado consistirá em porcentagem dos módulos e variação dos ângulos das correntes injetadas nas barras em relação às respectivas correntes na freqüência fundamental. Ou seja, para $n$ barras de estimação consideradas tem-se que a dimensão do indivíduo deve ser de $2 n$, correspondendo às $n$ porcentagens de módulo e às $\mathrm{n}$ variações de ângulos das correntes a serem estimadas.

Além das informações citadas, cada parâmetro de estimação do indivíduo possui também um fator que estabelece seu passo de mutação $\sigma$, o que representa a 'distância' que um indivíduo filho poderá estabelecer no espaço de soluções em relação à localização do indivíduo pai. Sendo assim, cada porcentagem de módulo e cada variação de ângulo possui um passo de mutação associado.

Como exemplo, a representação de um indivíduo genérico para a rede elétrica apresentada na Fig. 2, na qual se desejam estimar correntes harmônicas injetadas nas 3 barras da rede, pode ser realizada como segue:

$$
\text { Individuo }=\left\{\begin{array}{cccc}
k_{n}^{1} & \sigma_{k, n}^{1} & \phi_{n}^{1} & \sigma_{\phi, n}^{1} \\
k_{n}^{2} & \sigma_{k, n}^{2} & \phi_{n}^{2} & \sigma_{\phi, n}^{2} \\
k_{n}^{3} & \sigma_{k, n}^{3} & \phi_{n}^{3} & \sigma_{\phi, n}^{3}
\end{array}\right\}
$$

onde:

$k_{n}^{i}$ representa a porcentagem do módulo de corrente fundamental da barra $i$ para a ordem harmônica $n$.

$\phi_{n}^{i}$ representa a variação do ângulo de corrente fundamental da barra $i$ para a ordem harmônica $n$.

$\sigma_{k, n}^{i}$ representa o passo de mutação referente ao parâmetro $k$ da barra $i$ para a ordem harmônica $n$.

$\sigma_{\phi, n}^{i}$ representa o passo de mutação referente ao parâmetro $\phi$ da barra $i$ para a ordem harmônica $n$.

$I_{1}^{i} \mathbf{e} \theta_{1}^{i}$, apresentados na Fig. 2, representam os parâmetros corrente e ângulo de freqüência fundamental da barra $i$.

O número de indivíduos a ser considerado na população inicial consiste em um parâmetro empírico e deve ser avaliado de acordo com cada aplicação.

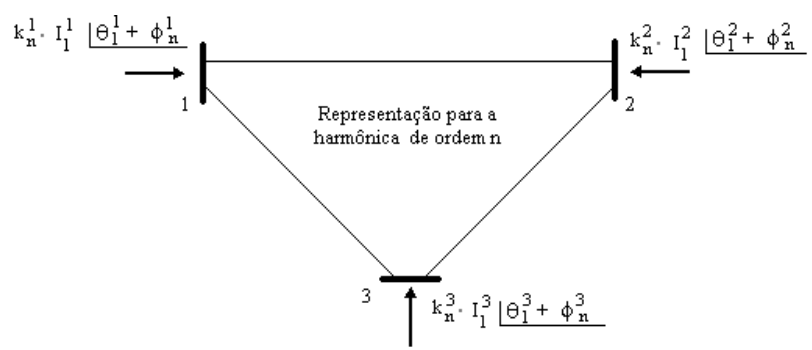

Figura 2: Representação dos indivíduos em uma ordem harmônica $n$. 


\subsection{Mutação}

Como descrito em Back et al. (1997), em Estratégias Evolutivas, a mutação tem um papel central no direcionamento da evolução de um único indivíduo. Sendo assim, cada indivíduo gera outros $n \_m u t a c a o$ indivíduos. As variações impostas por mutação são de pequeno passo em torno do indivíduo original.

Para melhorar os passos de mutação, foi introduzida a autoadaptação, conforme definida nas referências (Fogel, 1995; Schwefel, 1981). Esta visa otimizar o parâmetro $\sigma$ de desvios em relação à solução ou de passos de mutação em função da evolução da população de soluções de forma que, à medida que a população se aproxima da solução do problema, o passo de mutação diminua.

Matematicamente, pode-se definir a mutação com autoadaptação como:

$$
\begin{gathered}
\sigma_{i}^{\prime}=\sigma_{i} \cdot \exp \left(\tau^{\prime} \cdot N(0,1)+\tau \cdot N_{i}(0,1)\right) \\
x_{i}^{\prime}=x_{i}+\sigma_{i}^{\prime} \cdot N_{i}(0,1)
\end{gathered}
$$

onde:

$\sigma^{\prime}{ }_{i}$ é a variação do parâmetro $\sigma$ de índice $i$

$\sigma_{i}$ é o passo de mutação

$N_{i}(0,1)$ é o valor sorteado a cada geração com distribuição normal de média 0 e desvio padrão 1 .

$N(0,1)$ é o valor sorteado a cada geração com distribuição normal de média 0 e desvio padrão 1 . Este se mantém constante para todo indivíduo.

$\tau^{\prime}$ é a taxa de aprendizado $\left(\propto(\sqrt{2 \beta})^{-1}\right)$

$\tau$ é a taxa de aprendizado $\left(\propto(\sqrt{2 \sqrt{\beta}})^{-1}\right)$

Nesta modelagem, os parâmetros $\beta$ e $\sigma$ devem ser ajustados para cada aplicação, onde $\beta$ é uma constante que visa controlar a variabilidade da mutação.

\subsection{Recombinação}

O operador recombinação, aliado à mutação, tem por objetivo introduzir variabilidade à busca aleatória da solução do problema. Parte-se do princípio que a troca de informações "genéticas" entre indivíduos de uma mesma espécie pode resultar em indivíduos melhores ou, ainda, evitar que o algoritmo convirja para "ótimos" locais.
O cruzamento consiste em criar um novo indivíduo que contenha informação genética resultante da combinação das informações genéticas de seus pais.

Neste artigo, os parâmetros de cada indivíduo são gerados por um processo de reprodução por meio de duas regras, quais sejam média aritmética dos parâmetros e dos passos dos indivíduos pais ou troca das informações. Assim, os parâmetros porcentagem de módulo e variação de ângulo da corrente fundamental, que ocupam posições específicas na codificação do indivíduo, são modificados de acordo com uma dessas regras. A comparação de utilização destas duas regras para o problema de EEH é analisada no item 4.5.

\subsection{Avaliação}

A avaliação dos indivíduos da população deve indicar o quão perto da melhor solução está um indivíduo, considerando que cada indivíduo representa uma solução para o problema.

Uma vez que se pretendem estimar valores de distorções harmônicas em pontos de uma rede elétrica, sendo conhecidos os valores das distorções em pontos de medições, o procedimento de avaliação dos indivíduos da população medirá a proximidade dos valores de tensão propostos nos indivíduos dos valores medidos.

Para o problema em estudo, a função de avaliação será determinada a partir da soma dos erros médios quadráticos, conforme equações (6) e (7).

A nota de um determinado indivíduo nas estimações de distorções harmônicas para a frequiência $h$, que expressa sua avaliação na EE, deve ser obtida a partir dos erros de estimação de cada barra. Sendo assim, para um sistema de monitoramento de qualidade de energia com $n_{\text {med }}$ medições, foi adotada como a nota de um indivíduo o inverso da soma dos erros quadrados de estimação. Esta nota é expressa pela equação (12) que segue:

$$
\operatorname{Nota}_{h}=\frac{1}{\sum_{i=1}^{n_{\text {med }}}\left(e e_{h}^{i}\right)^{2}}
$$

Foram também considerados na avaliação valores que se situam fora do intervalo admitido para um indivíduo. Desta forma, indivíduos que apresentem erros superiores a determinado valor serão penalizados, obtendo notas baixas.

\subsection{Seleção}

No algoritmo de EE proposto, a operação de seleção dos indivíduos para a formação de gerações futuras é determinística, pois se limita a selecionar os melhores indivíduos em 


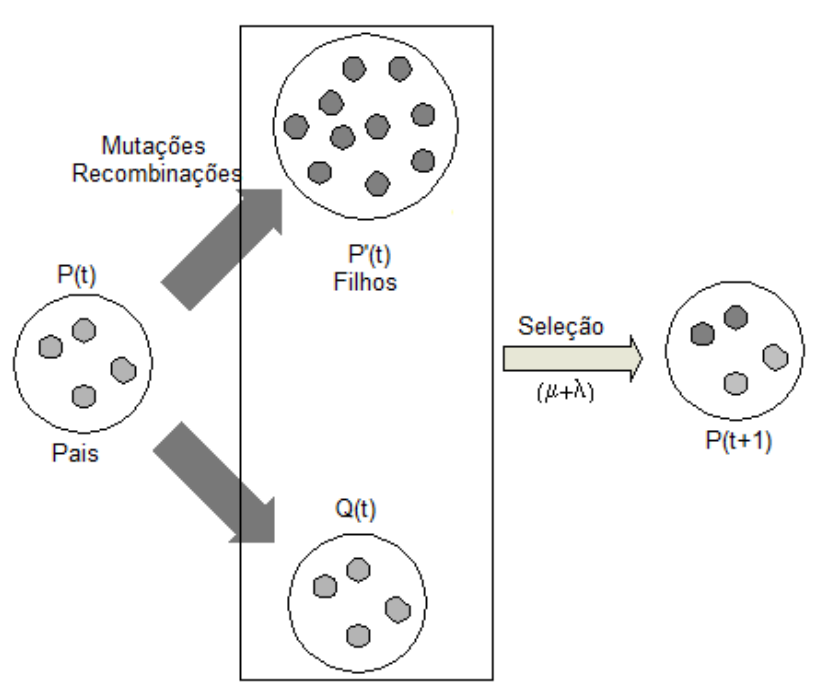

Figura 3: Método de seleção $(\mu+\lambda)$

um universo que pode ser "população inicial" mais "filhos" $(\mu+\lambda)$ ou somente "filhos" $(\mu, \lambda)$ (Fogel, 1995). A Fig.3 apresenta o método de seleção $(\mu+\lambda)$.

\section{AJUSTES NOS PARÂMETROS DO AL- GORITMO}

Antes de se iniciar uma estimação com o algoritmo baseado em EE, é necessário entender a sensibilidade dos resultados frente a variações nos parâmetros evolutivos já mencionados.

A rede de 14 barras apresentada na Fig. 4 foi utilizada nas análises de sensibilidade do algoritmo. Os dados desta rede, disponibilizados no Apêndice, foram obtidos do sítio do Institute of Electrical and Electronical Engineering (IEEE). Para os ajustes, foi considerada a componente harmônica de ordem 3 , e o número de medidores instalados na rede, indicados na figura, foi considerado fixo em 6 , nas barras $2,4,8$, 9,12 e 14 .

\subsection{Ajuste do Número de Indivíduos}

Como primeiro parâmetro de ajuste de um algoritmo de EE, apresenta-se o número de indivíduos na população inicial.

Nas avaliações, consideraram-se inicialmente fixos o número de gerações igual a 100, o número de mutações por indivíduo igual a 5, a taxa de recombinação por geração igual a $10 \%$, o passo de mutação inicial igual a 1 para módulos e $\pi$ para ângulos, o parâmetro $n$ referente à auto-adaptação igual a 2 , a forma de evolução $(\mu+\lambda)$ e o método de recombinação igual à média dos valores dos parâmetros.

Foram realizadas trinta simulações para as alternativas de [2

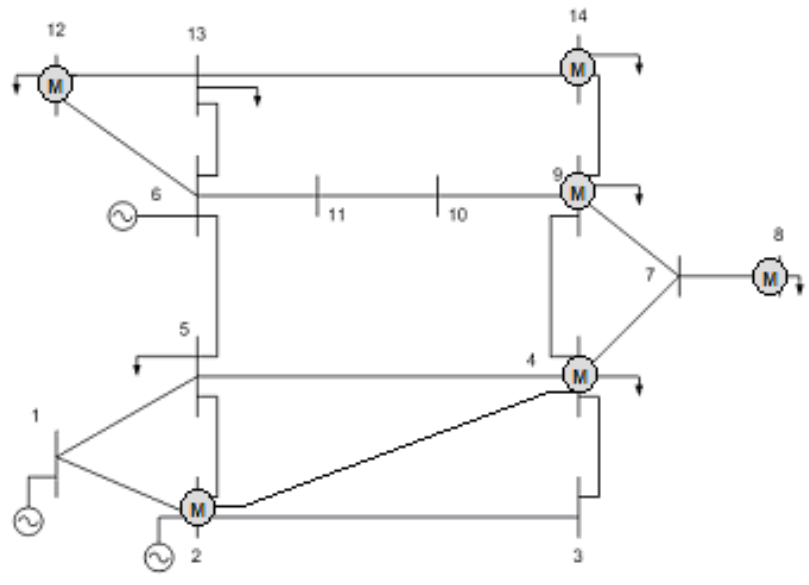

Figura 4: Rede elétrica utilizada nas avaliações.

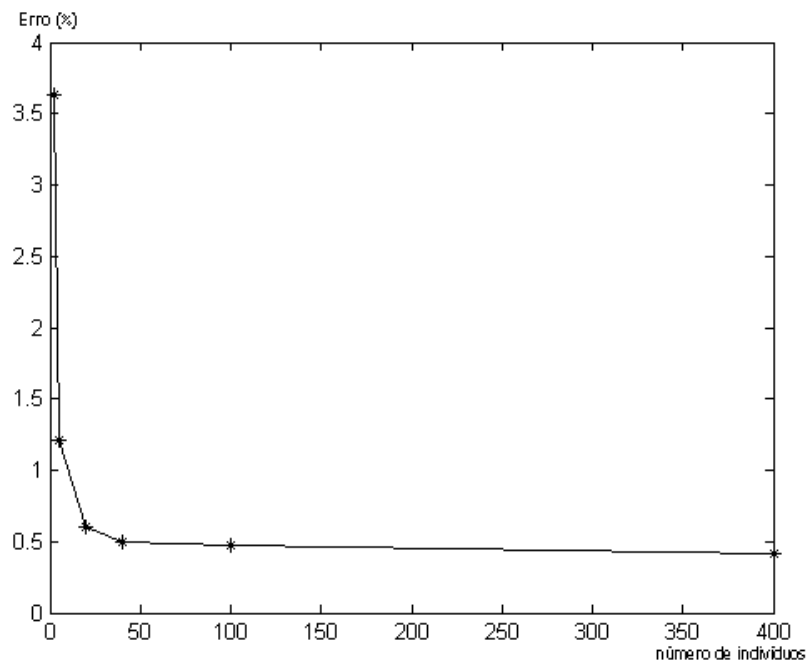

Figura 5: Erro absoluto médio de estimação em função do número de indivíduos na população inicial.

\section{0 ] indivíduos.}

Os resultados apresentados na Fig. 5 ilustram a média dos erros relativos de estimação em cada barra, para cada alternativa de indivíduos considerados na população inicial. Percebe-se que, à medida que é aumentado o número de indivíduos na população inicial, o erro na estimação diminui. Entretanto, a diminuição do erro a partir de 20 indivíduos não é muito significativa, tornando este número de indivíduos na população inicial suficiente para garantir uma boa estimação.

\subsection{Ajuste do Passo de Mutação}

O passo de mutação determina a distância entre o indivíduo pai e o indivíduo filho, estabelecendo onde este pode posicionar-se no espaço de soluções. Um passo muito grande 


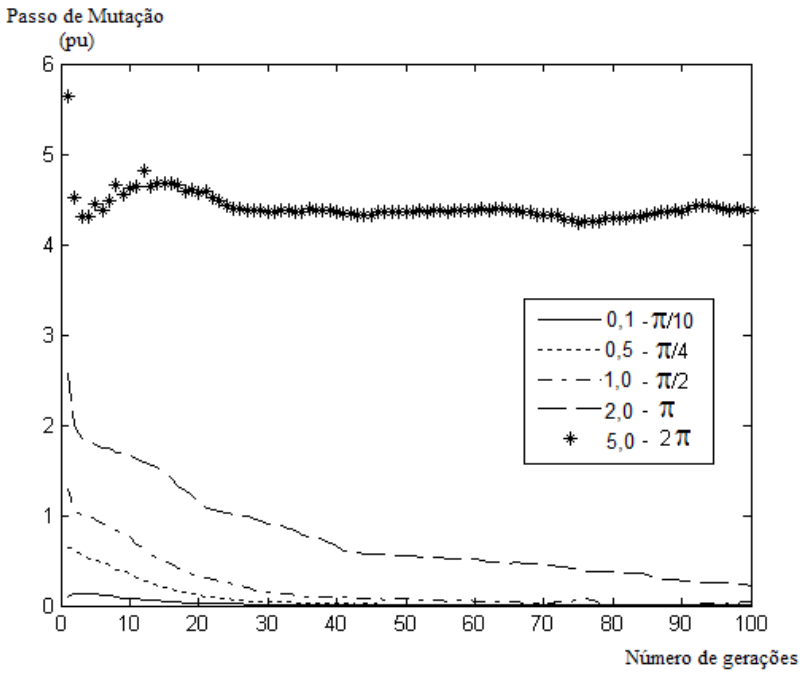

Figura 6: Evolução dos passos de mutação, combinação módulo + fase, para diferentes valores iniciais.

de mutação poderá introduzir muita variação nas populações de filhos, descaracterizando o conceito da mutação em EE. Já um passo de mutação muito pequeno implicaria variações muito pequenas nos indivíduos filhos, diminuindo, assim, a velocidade de convergência do algoritmo.

Para as porcentagens de módulo, foram analisados os valores $\left[\begin{array}{llll}0,1 & 0,5 & 1 & 2\end{array}\right]$ e, para as variações de ângulos, os valores $[\pi / 10 \pi / 4 \pi / 2 \pi 2 \pi]$, como passos de mutações iniciais. As análises foram realizadas utilizando as mesmas configurações empregadas para os ajustes de número de indivíduos, sendo este fixado em 20. Para cada configuração de passo de mutação, foram simulados 30 casos.

A Fig. 6 apresenta a evolução dos passos de mutação para os valores iniciais considerados. Percebe-se que valores iniciais muito altos para os passos de mutação implicam menor velocidade de convergência, dificultando a obtenção de bons resultados de estimação. Por outro lado, a escolha de valores iniciais muito baixos convergem rapidamente para zero, podendo eliminar a variabilidade na evolução e dificultar o alcance de um ótimo global. O par de valores medianos 1 para porcentagens de módulos e $\pi / 2$ para variações de ângulos representam uma configuração que atende as necessidades do algoritmo de EEH.

\subsection{Ajuste do Número de Mutações por Indivíduo}

Outro parâmetro importante para ajustar é o número de mutações que cada indivíduo de uma população de pais pode sofrer. Este parâmetro está ligado às características do espaço de soluções, uma vez que quanto maior for este parâ- metro, maior será a cobertura do espaço. Foram escolhidos para análise os valores [1 51050 100] como número de mutações. Nestas simulações, o número de indivíduos foi fixado em 20 e os passos de mutações fixados em 1 para as porcentagens de módulos, e em $\pi / 2$ para as variações de ângulos. Foram realizadas 30 simulações para cada caso considerado.

Segundo apresentado na Tabela I, o erro de estimação tende a diminuir com o aumento do número de mutações por indivíduo até um certo limite, em que os resultados deixam de ser tão dependentes deste parâmetro. Como exemplo, percebese que, variando de 50 para 100 o número de mutações por indivíduo, o erro na estimação melhora nas barras com medição (em destaque cinza na Tabela I), porém pode piorar nas barras sem medição. Outro ponto primordial na escolha do número de mutações por indivíduo em cada geração está em sua relação direta com o aumento no tempo de simulação. Ou seja, quanto maior o número de mutações, maior o tempo de simulação.

Tabela I: Erro médio percentual para uma dada ordem harmônica em função do número de mutações por indivíduo em cada geração.

\begin{tabular}{|c|c|c|c|c|c|}
\hline \multirow{2}{*}{ Barra } & \multicolumn{5}{|c|}{ NÚMERO DE MUTAC̃ÕES POR INDIVÍDUO } \\
\cline { 2 - 6 } & 1 & 5 & 10 & 50 & 100 \\
\hline 1 & 1,3526 & 0,6649 & 0,5273 & 0,4022 & 0,4516 \\
\hline 2 & 1,3817 & 0,4989 & 0,4346 & 0,2562 & 0,1543 \\
\hline 3 & 1,2358 & 0,3120 & 0,1531 & 0,0842 & 0,0589 \\
\hline 4 & 1,1489 & 0,5558 & 0,5385 & 0,3228 & 0,2269 \\
\hline 5 & 1,1660 & 0,6300 & 0,6175 & 0,3720 & 0,3776 \\
\hline 6 & 1,4802 & 1,1147 & 0,9023 & 0,8349 & 0,9120 \\
\hline 7 & 0,8732 & 0,2931 & 0,2621 & 0,0993 & 0,0688 \\
\hline 8 & 1,2676 & 0,4537 & 0,3389 & 0,2006 & 0,1239 \\
\hline 9 & 1,2049 & 0,6523 & 0,5334 & 0,2726 & 0,1551 \\
\hline 10 & 1,3017 & 1,0191 & 0,7979 & 0,5766 & 0,8354 \\
\hline 11 & 1,1962 & 0,9638 & 1,0658 & 0,9433 & 1,2052 \\
\hline 12 & 1,5128 & 0,4517 & 0,2922 & 0,0942 & 0,0514 \\
\hline 13 & 1,7985 & 0,9765 & 0,6746 & 0,7539 & 0,9224 \\
\hline 14 & 1,5170 & 0,5370 & 0,4112 & 0,1886 & 0,0953 \\
\hline
\end{tabular}

\subsection{Ajuste da Auto-Adaptação}

Como apresentado em Back et al. (1997), a auto-adaptação insere na evolução do indivíduo uma correção de seu passo de mutação. Assim, o passo de mutação deve diminuir à medida que o indivíduo se aproxima do "ótimo". O comportamento da mutação frente às mudanças nos parâmetros da 
auto-adaptação pode mudar em sua forma de evolução e velocidade de evolução.

A utilização de um passo de mutação para cada parâmetro do indivíduo fornece maior diversidade na evolução, permitindo aos indivíduos evoluir para regiões elípticas em torno do indivíduo pai (Back et al., 1997).

O parâmetro $\beta$ está relacionado à velocidade de diminuição do passo a cada geração. Este parâmetro foi avaliado para os valores [0,1 1210 100], sendo rodados 30 casos para cada análise.

Apesar de o esperado teórico relacionado às variações no parâmetro $\beta$ terem sido expressivos, não foram percebidos benefícios diretos na variação dos valores de $\beta$ maiores que 1 . Para valores menores que 1 , foram verificadas variações expressivas na evolução dos passos de mutação, como pode ser verificado na Fig. 7, que apresenta a evolução dos passos de mutação para os valores de $\beta$ considerados. A curva com maior variação nesta figura representa $\beta$ igual a 0,1 . Percebese que, para os demais valores de $\beta$ considerados, a evolução dos passos de mutação foram similares.

Embora se tenha observado a grande variação apresentada na Fig. 7, os erros na estimação não sofreram variações expressivas em função da modificação deste parâmetro. A Tabela II apresenta os erros de estimação em cada barra do sistema em estudo para cada valor do parâmetro $\beta$ considerado. Pela análise dos erros e dos demais parâmetros pré-fixados para análise, não se pode definir um valor ótimo para o parâmetro $\beta$.

\subsection{Ajuste do Operador Recombinação}

A introdução na evolução de variações além das possíveis por meio das mutações pode ser realizada, conforme já mencionado, por meio do operador recombinação, pela média dos parâmetros ou troca das informações entre dois indivíduos. Esta operação genética tende a criar indivíduos capazes de redirecionar a população em evolução, evitando a convergência do algoritmo em ótimos locais.

As duas alternativas de recombinação citadas foram analisadas, tendo sido rodados 100 casos para cada uma.

Conforme apresentado na Fig. 8, a utilização das médias dos parâmetros como forma de evolução obteve um comportamento com maiores variações, frente à utilização da troca de informações entre os indivíduos pais. Embora esta variabilidade na evolução dos passos de mutação não tenha correspondido em grandes variações nos erros de estimação, como apresentados na Tabela III, espera-se que o algoritmo tenha maior dificuldade de convergência para ótimos locais, utilizando a média dos parâmetros como forma de evolução.
Tabela II: Erro de estimação em função do parâmetro $\beta$ de auto-adaptação.

\begin{tabular}{|c|c|c|c|c|c|}
\hline \multirow{2}{*}{ Barra } & \multicolumn{5}{|c|}{ Valor do parâmetro $\beta$ na auto-adaptação } \\
\cline { 2 - 6 } & 0,1 & 1 & 2 & 10 & 100 \\
\hline 1 & 0,5085 & 0,5952 & 0,7417 & 0,5734 & 0,7430 \\
\hline 2 & 0,4007 & 0,3644 & 0,5359 & 0,4019 & 0,4455 \\
\hline 3 & 0,3340 & 0,2444 & 0,3433 & 0,2259 & 0,2638 \\
\hline 4 & 0,5242 & 0,4556 & 0,5093 & 0,5000 & 0,5638 \\
\hline 5 & 0,5556 & 0,4913 & 0,5639 & 0,5547 & 0,5550 \\
\hline 6 & 1,1131 & 1,3904 & 1,1678 & 1,4486 & 1,1684 \\
\hline 7 & 0,3571 & 0,3140 & 0,2545 & 0,2709 & 0,2964 \\
\hline 8 & 0,6394 & 0,5066 & 0,5088 & 0,5951 & 0,4053 \\
\hline 9 & 0,6866 & 0,7909 & 0,6228 & 0,5632 & 0,5148 \\
\hline 10 & 1,0471 & 1,3505 & 1,0594 & 1,0291 & 0,8784 \\
\hline 11 & 0,9901 & 1,3673 & 1,1234 & 1,3224 & 1,0632 \\
\hline 12 & 0,4204 & 0,4734 & 0,5121 & 0,4150 & 0,5011 \\
\hline 13 & 1,0604 & 0,9386 & 0,8408 & 0,9563 & 1,0008 \\
\hline 14 & 0,6008 & 0,6326 & 0,5012 & 0,4886 & 0,5825 \\
\hline
\end{tabular}

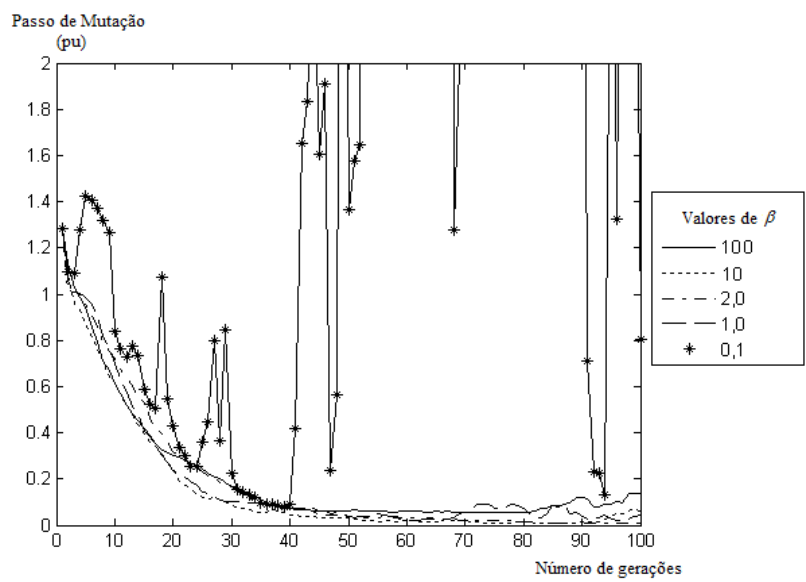

Figura 7: Evolução dos passos de mutação em função do parâmetro $\beta$.

\subsection{Ajuste da Forma de Evolução}

Conforme apresentado na referência Back and Schwefel (1996), não há consenso em relação à forma de evolução. Neste trabalho, as formas $(\mu+\lambda)$ e $(\mu, \lambda)$ foram avaliadas, tendo sido simulados 100 casos por análise.

A Fig. 9 apresenta a evolução dos passos de mutação para as duas formas de evolução consideradas. A forma de evolução $(\mu, \lambda)$, que se caracteriza por não apresentar elitismo, gerou 


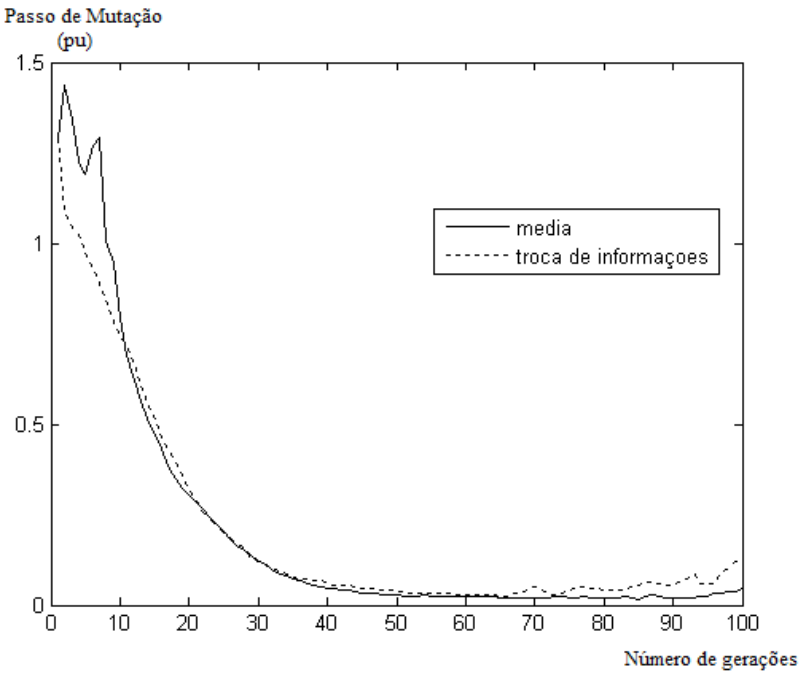

Figura 8: Evolução dos passos de mutação em função da forma de recombinação.

grandes variações nos passos de mutação, não convergindo com o passar das gerações de evolução.

Pode-se verificar a não convergência desta forma de evolução por meio dos erros de estimação apresentados na Tabela IV.

Tabela III: Erro relativo na estimação em função da forma de recombinação.

\begin{tabular}{|c|c|c|}
\hline \multirow{2}{*}{ Barra } & \multicolumn{2}{|c|}{$\begin{array}{r}\text { ERRO EM FUNÇÃO DA FORMA } \\
\text { DE RECOMBINAÇÃO }\end{array}$} \\
\cline { 2 - 3 } & Média & Troca de Informações \\
\hline 1 & 0,8319 & 0,8144 \\
\hline 2 & 0,6432 & 0,5845 \\
\hline 3 & 0,3528 & 0,3748 \\
\hline 4 & 0,6327 & 0,5827 \\
\hline 5 & 0,6430 & 0,6139 \\
\hline 6 & 1,1738 & 1,3238 \\
\hline 7 & 0,4024 & 0,3380 \\
\hline 8 & 0,5437 & 0,6643 \\
\hline 9 & 0,7770 & 0,6815 \\
\hline 10 & 1,2743 & 1,1689 \\
\hline 11 & 1,1813 & 1,0825 \\
\hline 12 & 0,5382 & 0,6244 \\
\hline 13 & 0,8523 & 0,9375 \\
\hline 14 & 0,6936 & 0,6687 \\
\hline
\end{tabular}

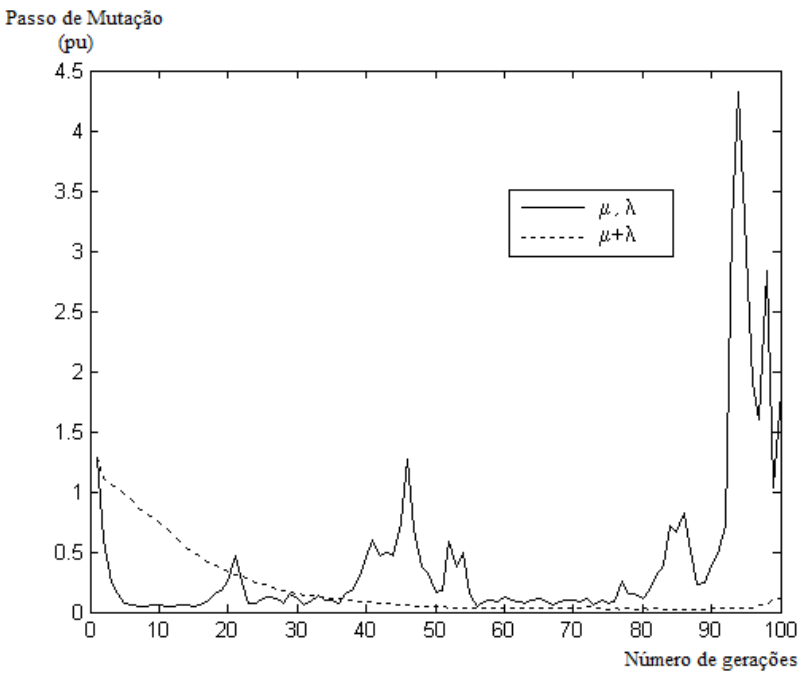

Figura 9: Evolução dos passos de mutação em função da forma de evolução: $(\mu+\lambda)$ e $(\mu, \lambda)$.

Tabela IV: Erro de estimação em função da forma de evolução.

\begin{tabular}{|c|c|c|}
\hline \multirow{2}{*}{ Barra } & \multicolumn{2}{|c|}{ FORMA DE EVOLUÇÃO } \\
\cline { 2 - 3 } & $(\mu, \lambda)$ & $(\mu+\lambda)$ \\
\hline 1 & 14,7769 & 0,8454 \\
\hline 2 & 14,6325 & 0,6937 \\
\hline 3 & 14,4508 & 0,4865 \\
\hline 4 & 13,4392 & 0,7645 \\
\hline 5 & 13,6969 & 0,7716 \\
\hline 6 & 11,8724 & 1,2729 \\
\hline 7 & 11,4144 & 0,4601 \\
\hline 8 & 11,2406 & 0,5736 \\
\hline 9 & 10,6349 & 0,7973 \\
\hline 10 & 10,3667 & 1,1879 \\
\hline 11 & 10,5926 & 1,2099 \\
\hline 12 & 10,8155 & 0,6366 \\
\hline 13 & 10,5179 & 1,1006 \\
\hline 14 & 9,4858 & 0,7107 \\
\hline
\end{tabular}

\section{RESULTADOS}

A rede elétrica apresentada na Fig. 4 foi utilizada na avaliação do algoritmo proposto, a fim de estimar a Distorção Harmônica Total (DHT) em cada barra da rede. Com medidores instalados nas barras 2, 4, 8, 9, 12 e 14, foram consideradas as ordens harmônicas 3, 5, 7, 9, 11 e 13 na composição da DHT em cada barra do sistema. Como mencionado, o 
algoritmo proposto efetua a estimação de cada componente harmônica de forma independente. Em seguida, o cálculo da distorção harmônica total é efetuado. O algoritmo foi todo implementado em MATLAB, envolvendo rotinas para o fluxo de potência na freqüência fundamental, o fluxo de potência para cada ordem harmônica e a estratégia evolutiva propriamente dita. Como configurações do algoritmo foram consideradas:

- Número de gerações igual 500;

- Número de indivíduos na população inicial igual a 40;

- Número de mutações por indivíduo igual a 5;

- Taxa de recombinação por geração igual a 10\%;

- Passo de mutação inicial igual a 1 para módulos e $\pi$ para ângulos;

- $\beta$ referente à auto-adaptação igual a 2;

- Evolução $(\mu+\lambda)$;

- Método de recombinação igual à média.

A Tabela V apresenta os erros de estimação para as ordens harmônicas consideradas individualmente. Esses resultados representam uma média dos erros para os 30 casos rodados para cada ordem harmônica. Resultados semelhantes foram publicados na referência (Kagan and Arruda, 2006), embora com foco na estimação de uma ordem harmônica específica. Verifica-se, nesta tabela, que os valores de erros de estimação para as ordens harmônicas individualmente são muito baixos. As linhas em destaque na tabela representam as barras com medição.

A Fig. 10 apresenta a nota média dos melhores indivíduos para cada ordem harmônica. Esta nota é obtida por meio do inverso da soma dos desvios quadrados em todas as barras com medição, conforme equação (12); é computada a cada geração do algoritmo e indica a velocidade de evolução no espaço de resultados. Embora em algumas ordens harmônicas os melhores indivíduos tenham obtido notas superiores a outras ordens, os erros de estimação foram satisfatórios, como pode ser observado na Tabela $\mathrm{V}$ apresentada.

O crescimento continuado das notas dos indivíduos em todas as ordens harmônicas confirma o elitismo do algoritmo proposto e sua capacidade de encontrar uma solução para o problema. O crescimento monótono das notas dos melhores indivíduos em alguns casos pode representar uma dificuldade do operador recombinação em imputar uma variabilidade aos indivíduos que os leve mais rapidamente à região de melhor solução do problema, o que está ligado à complexidade do espaço de soluções.
Tabela V: Erro de estimação (\%) para as ordens harmônicas consideradas individualmente.

\begin{tabular}{|c|c|c|c|c|c|c|}
\hline \multirow{2}{*}{ Barra } & \multicolumn{7}{|c|}{ Ordens Harmônicas } \\
\cline { 2 - 7 } & 3 & 5 & 7 & 9 & 11 & 13 \\
\hline 1 & 0,3011 & 1,4361 & 0,9143 & 0,2239 & 0,0416 & 0,5515 \\
\hline 2 & 0,2087 & 1,4178 & 0,2884 & 0,0352 & 0,0265 & 0,4715 \\
\hline 3 & 0,1155 & 1,3434 & 0,1728 & 0,0205 & 0,0213 & 0,4600 \\
\hline 4 & 0,2059 & 1,0616 & 0,0411 & 0,0053 & 0,0017 & 0,0734 \\
\hline 5 & 0,3408 & 1,1694 & 0,2054 & 0,2230 & 0,0404 & 0,4252 \\
\hline 6 & 0,9816 & 0,7969 & 0,1441 & 0,6052 & 0,5011 & 0,5294 \\
\hline 7 & 0,0782 & 0,6707 & 0,0535 & 0,0125 & 0,0875 & 0,2008 \\
\hline 8 & 0,1786 & 1,1199 & 0,1492 & 0,0124 & 0,0810 & 0,1084 \\
\hline 9 & 0,2241 & 0,5227 & 0,1913 & 0,0304 & 0,1671 & 0,4033 \\
\hline 10 & 0,1606 & 0,5286 & 0,5390 & 0,2800 & 0,5236 & 1,0011 \\
\hline 11 & 0,8894 & 0,5185 & 0,2987 & 0,3013 & 0,4648 & 0,7420 \\
\hline 12 & 0,0750 & 0,2000 & 0,0556 & 0,0250 & 0,0414 & 0,2551 \\
\hline 13 & 0,7392 & 0,2835 & 0,1388 & 0,2131 & 0,1332 & 0,7624 \\
\hline 14 & 0,0842 & 0,3115 & 0,3637 & 0,0717 & 0,1189 & 0,6046 \\
\hline & & & & & & \\
\hline
\end{tabular}
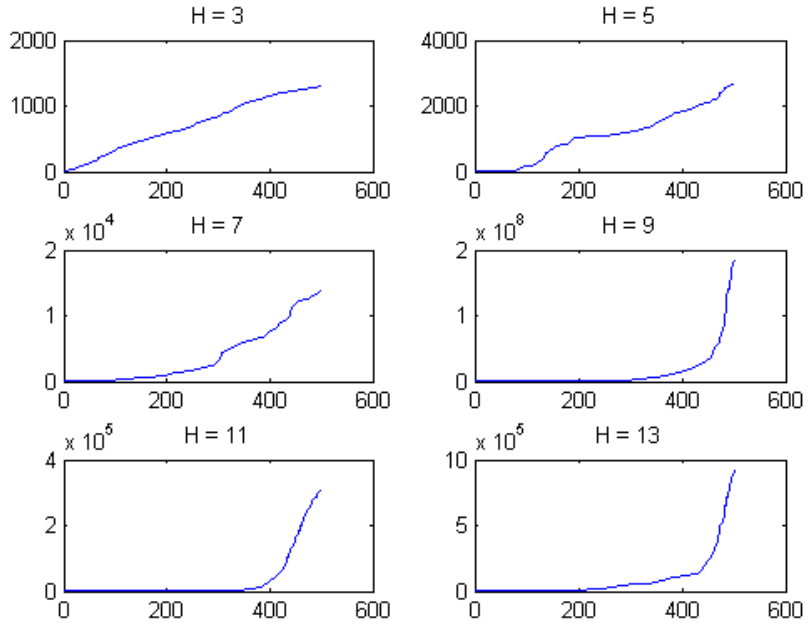

Figura 10: Nota média dos melhores indivíduos (eixo das ordenadas) para toda ordem harmônica em função das gerações (eixo das abscissas).

A Fig. 11 apresenta a evolução dos passos de mutação para cada ordem harmônica considerada. Percebe-se que, apesar de variações avulsas nos passos de mutação dos indivíduos em determinadas gerações, estes evoluem para valores próximos de zero. 

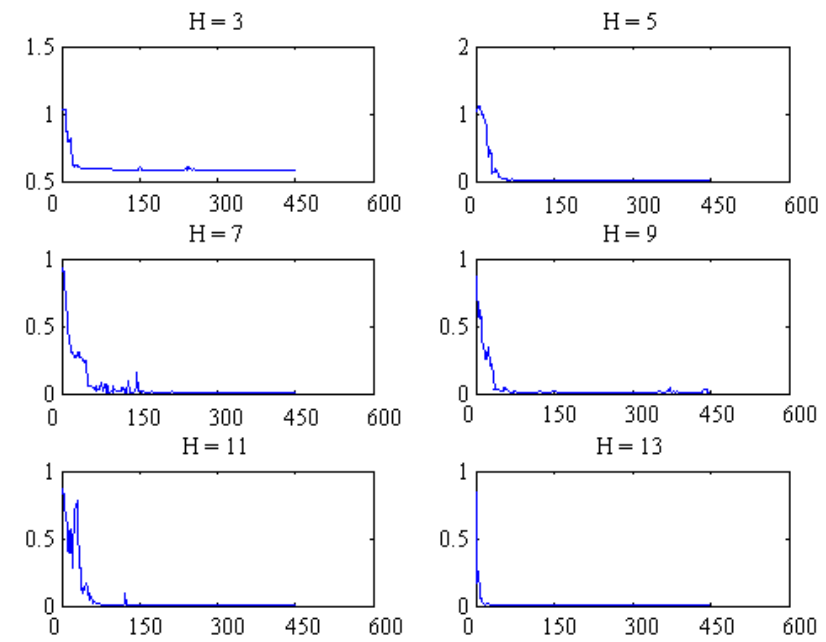

Figura 11: Evolução dos passos de mutação (eixo das ordenadas) para cada ordem harmônica $(\mathrm{H})$ em função das gerações (eixo das abscissas).

As variações encontradas na evolução dos passos de mutação representam a escolha de indivíduos, frutos de recombinações inserindo variabilidade na evolução.

Uma vez obtidos os valores de tensão para cada ordem harmônica considerada em cada barra do sistema, a DHT é obtida.

Desta forma, obtêm-se os valores apresentados na Tabela VI, onde observam-se os valores de DHT, de referência e estimados, para cada barra do sistema teste, bem como os erros de estimação, relativo e absoluto. Os valores de DHT de referência simulam os valores a serem encontrados.

O erro absoluto apresentado na Tabela VI representa o desvio obtido em relação à tensão nominal do sistema em estudo. Entretanto, é esperado que os erros em relação aos valores de THD (erros relativos) sejam muito superiores. Na quarta coluna da tabela em análise, percebe-se que esses erros ainda assim são baixos, apresentando um valor máximo inferior a $5 \%$.

Como forma de verificar a robustez da metodologia proposta, foram considerados medidores somente nas barras 2,8 e 12 do sistema. A Tabela VII apresenta os valores obtidos de DHT e erros de estimação considerando os três medidores instalados no sistema. Os elevados erros em algumas barras sem medição no sistema são decorrentes da pouca visibilidade dos medidores. Por outro lado, ainda com pouca visibilidade por parte do sistema de medição, os resultados podem ser considerados satisfatórios, tomando o erro absoluto (obtido, dividindo o erro relativo pelo valor do módulo da tensão na freqüência fundamental) como parâmetro. Este resultado se deve à topologia da rede.
Tabela VI: Valores de DHT de referência e calculados e erros relativos e absolutos de estimação.

\begin{tabular}{|c|c|c|c|c|}
\hline \multirow{2}{*}{ Barra } & \multicolumn{2}{|c|}{ DHT } & \multicolumn{2}{c|}{ Erro (\%) } \\
\cline { 2 - 5 } & referência & calculados & relativo & Absoluto \\
\hline 1 & 0,0545 & 0,0548 & 0,5468 & 0,0298 \\
\hline 2 & 0,0528 & 0,0529 & 0,1370 & 0,0072 \\
\hline 3 & 0,0502 & 0,0496 & 1,1816 & 0,0593 \\
\hline 4 & 0,0405 & 0,0395 & 2,4706 & 0,1001 \\
\hline 5 & 0,0421 & 0,0410 & 2,7515 & 0,1159 \\
\hline 6 & 0,0378 & 0,0379 & 0,3151 & 0,0119 \\
\hline 7 & 0,0394 & 0,0391 & 0,7450 & 0,0294 \\
\hline 8 & 0,0426 & 0,0424 & 0,2744 & 0,0117 \\
\hline 9 & 0,0409 & 0,0407 & 0,6056 & 0,0248 \\
\hline 10 & 0,0407 & 0,0425 & 4,4646 & 0,1818 \\
\hline 11 & 0,0398 & 0,0406 & 1,8083 & 0,0721 \\
\hline 12 & 0,0387 & 0,0388 & 0,2835 & 0,0110 \\
\hline 13 & 0,1952 & 0,2009 & 2,8884 & 0,5639 \\
\hline 14 & 0,2082 & 0,2085 & 0,1322 & 0,0275 \\
\hline
\end{tabular}

Tabela VII: Valores de DHT de referência e calculados e erros relativos e absolutos de estimação, considerando apenas 3 medidores instalados no sistema.

\begin{tabular}{|c|c|c|c|c|}
\hline \multirow{2}{*}{ Barra } & \multicolumn{2}{|c|}{ DHT } & \multicolumn{2}{c|}{ Erro (\%) } \\
\cline { 2 - 5 } & referência & calculados & relativo & Absoluto \\
\hline 1 & 0,0545 & 0,0560 & 2,7401 & 0,1493 \\
\hline 2 & 0,0528 & 0,0532 & 0,6888 & 0,0364 \\
\hline 3 & 0,0502 & 0,0481 & 4,0441 & 0,2028 \\
\hline 4 & 0,0405 & 0,0390 & 3,8868 & 0,1576 \\
\hline 5 & 0,0421 & 0,0431 & 2,2602 & 0,0952 \\
\hline 6 & 0,0378 & 0,0428 & 13,3189 & 0,5035 \\
\hline 7 & 0,0394 & 0,0396 & 0,4340 & 0,0171 \\
\hline 8 & 0,0426 & 0,0408 & 4,1465 & 0,1765 \\
\hline 9 & 0,0409 & 0,0438 & 7,1387 & 0,2921 \\
\hline 10 & 0,0407 & 0,0461 & 13,1931 & 0,5372 \\
\hline 11 & 0,0398 & 0,0449 & 12,5873 & 0,5016 \\
\hline 12 & 0,0387 & 0,0370 & 4,4007 & 0,1705 \\
\hline 13 & 0,0390 & 0,0401 & 2,5793 & 0,1007 \\
\hline 14 & 0,0416 & 0,0573 & 37,6589 & 1,5682 \\
\hline
\end{tabular}

Uma forma de melhorar os resultados de estimação é inserir, no algoritmo, outros dados conhecidos ou medidos do sis- 
tema em estudo, como, por exemplo, dados de medição de corrente. Já que a metodologia proposta considera as variações de ângulos e porcentagens de módulos da corrente como indivíduos das populações da estratégia evolutiva, a inserção desta informação restringe o espaço de busca do algoritmo, o que tende a levar a uma solução mais próxima do ótimo global.

A Tabela VIII apresenta os erros de estimação, considerando 3 medidores de tensão, como no exemplo anterior, e um medidor de injeção de corrente harmônica na barra 8 do sistema. Verifica-se, então, considerável redução dos erros de estimação na maioria das barras do sistema.

Tabela VIII: Valores de DHT de referência e calculados e erros relativos e absolutos de estimação, considerando 3 medidores de tensão e um de corrente.

\begin{tabular}{|c|c|c|c|c|}
\hline \multirow{2}{*}{ Barra } & \multicolumn{2}{|c|}{ DHT $(\%)$} & \multicolumn{2}{c|}{ Erro (\%) } \\
\cline { 2 - 5 } & referência & calculados & relativo & absoluto \\
\hline 1 & 0,0545 & 0,0542 & 0,4897 & 0,0267 \\
\hline 2 & 0,0528 & 0,0527 & 0,2176 & 0,0115 \\
\hline 3 & 0,0502 & 0,0486 & 3,1340 & 0,1572 \\
\hline 4 & 0,0405 & 0,0384 & 5,3272 & 0,2159 \\
\hline 5 & 0,0421 & 0,0403 & 4,3416 & 0,1830 \\
\hline 6 & 0,0378 & 0,0381 & 0,7013 & 0,0265 \\
\hline 7 & 0,0394 & 0,0380 & 3,4948 & 0,1378 \\
\hline 8 & 0,0426 & 0,0426 & 0,0602 & 0,0026 \\
\hline 9 & 0,0409 & 0,0404 & 1,2209 & 0,0500 \\
\hline 10 & 0,0407 & 0,0404 & 0,7511 & 0,0306 \\
\hline 11 & 0,0398 & 0,0386 & 3,1834 & 0,1268 \\
\hline 12 & 0,0387 & 0,0390 & 0,7811 & 0,0303 \\
\hline 13 & 0,0390 & 0,0443 & 13,5514 & 0,5291 \\
\hline 14 & 0,0416 & 0,0499 & 19,8447 & 0,8264 \\
\hline
\end{tabular}

\section{CONCLUSÕES}

Foi apresentada, neste trabalho, a estimação da distorção harmônica, individual e total, em barras de um sistema elétrico, a partir de medições em algumas barras. A técnica de Estratégias Evolutivas foi utilizada como ferramenta de otimização do problema.

$\mathrm{O}$ algoritmo proposto partiu de dados de medição de tensões harmônicas em algumas barras do sistema, com o propósito de estimar o conteúdo de DHT nas demais barras do sistema.

Os indivíduos considerados nesta metodologia são compostos por fatores (porcentagem do módulo e variação de ân- gulo) que representam a corrente harmônica, em uma barra em função da corrente fundamental na própria barra. A partir destes indivíduos, os quais definem as correntes harmônicas injetadas nas barras do sistema, os valores das tensões harmônicas são calculados, com a utilização da matriz de admitâncias da rede para a ordem harmônica considerada. A melhor solução para o problema consiste na distribuição de tensões calculadas, que minimiza o erro quadrático entre os valores calculados e os valores medidos. Este procedimento é repetido para cada ordem harmônica de interesse e, finalmente, o valor da DHT é calculado.

Uma rede de 14 barras, cujos dados foram obtidos no sítio do (IEEE), foi utilizada nas simulações.

Foram apresentados os resultados da estimação individual das tensões harmônicas, considerando as ordens $3^{a}, 5^{a}, 7^{a}$, $9^{a}, 11^{a}$ e $13^{a}$. Foram simulados 30 casos para cada ordem harmônica e o algoritmo mostrou-se robusto e com excelentes resultados de estimação.

As distorções estimadas individualmente foram utilizadas para obter a DHT em cada barra. Os resultados obtidos apresentaram erros inferiores a $1 \%$ em todas as simulações.

Foram também abordadas formas de diminuição de erros de estimação em situações de pouca visibilidade por parte do sistema de medição, sendo estas o conhecimento de correntes harmônicas em linhas ou em cargas em pontos da rede em estudo ou informações extras de medição.

Utilizando apenas 3 medidores de tensões harmônicas instalados na rede e os valores de correntes harmônicas em uma das barras, o algoritmo foi capaz de diminuir erros de estimação em mais de $50 \%$ em barras próximas dessas medições, quando comparado com a análise desconsiderando as medições de corrente.

Salienta-se que os resultados obtidos neste trabalho, embora tenham focado uma rede elétrica de pequeno porte, indicam um vasto campo de análise quanto à EEH.

\section{REFERÊNCIAS}

Back, T. and Schwefel, H.-P. (1996), Evolutionary Computation: An Overview, Proceedings of IEEE International Conference on Evolutionary Computation: 20-29.

Back, T., Hammel, U. and Schwefel, H.-P. (1997). Evolutionary Computation: Comments on the History and Current State, IEEE Transactions on Evolutionary Computation 1: 3-17.

Du, Z. P., Arrilaga, J., Watson, N. R. and Chen, S. (1999). Identification of Harmonic Sources of Power systems 
Using State Estimation, IEE Proceedings on Generation, Transmission and Distribution 146: 7-12.

Du, Z. P., Arrilaga, J., Watson, N. R. and Chen, S. (1998). Implementation of Harmonic State Estimation, Proceedings of the 8th International Conference on Harmonics and Quality of Power 1: 273-278.

Fogel, D. B. (1995). Evolutionary computation: toward a new philosophy of machine intelligence. Piscataway, NJ: IEEE Press.

Heydt, G. T. (1989). Identification of Harmonic Sources by a State Estimation Technique, IEEE Transactions on Power Delivery 4: 8.

IEEE, Test Systems for Harmonics Modeling and Simulation, in Task Force on Harmonics Modeling and Simulation, IEEE Power Engineering Society, http://grouper.ieee.org/groups / harmonic/simulate/download.htm.

Kagan, N. and Arruda, E. F. (2006). Harmonic Estimation Using Evolutionary Algorithms, ICHQP - International Conference on Harmonics and Quality of Power.

Meliopoulos, A. P. S., Zhang, F. and Zelingher, S. (1992) Hardware and Software Requirements for a Transmission System Harmonic Measurement System, Harmonics in Power Systems, ICHPSV: 330-338.

Meliopoulos, A. P. S., Zhang, F. and Zelingher, S. (1994). Power System Harmonic State Estimation, IEEE Transactions on Power Delivery 9: 1701-1709.

Schwefel, H.-P. (1981). Numerical optimization of computer models. Chichester; New York: John Wiley \& Sons.

\section{APÊNDICE}

Nas tabelas A.1 e A.2 são apresentados os dados de ligações e barras, na freqüência fundamental, para a rede da figura 4. Os dados em pu estão referidos à base 100 MVA.

Nas tabelas A.3, A.4 e A.5 são fornecidas as injeções de correntes para as ordens harmônicas $3,5,7,9,11$ e 13, perseguidas pelos algoritmo de EE, e que produzem as DHTs de tensão tomadas como referência para validação da metodologia..
Tabela A.1 - Dados de ligações (LT - linha de transmissão e TR transformadores)

\begin{tabular}{|c|c|c|c|c|c|}
\hline $\begin{array}{l}\text { Nó } \\
\text { inicial }\end{array}$ & $\begin{array}{l}\text { Nó } \\
\text { final }\end{array}$ & Tipo & $\begin{array}{l}\text { Resistência } \\
\quad(\mathrm{pu})\end{array}$ & $\begin{array}{c}\text { Reatância } \\
\text { (pu) }\end{array}$ & $\begin{array}{l}\text { Susceptância } \\
\text { Capacitiva } \\
\text { (pu) }\end{array}$ \\
\hline 4 & 7 & TR & 0 & 0,209 & 0 \\
\hline 4 & 9 & TR & 0 & 0,556 & 0 \\
\hline 5 & 6 & TR & 0 & 0,250 & 0 \\
\hline 6 & 11 & LT & 0,095 & 0,199 & 0 \\
\hline 6 & 12 & LT & 0,123 & 0,256 & 0 \\
\hline 6 & 13 & $\mathrm{LT}$ & 0,066 & 0,130 & 0 \\
\hline 7 & 8 & $\mathrm{TR}$ & 0 & 0,176 & 0 \\
\hline 7 & 9 & TR & 0 & 0,110 & 0 \\
\hline 9 & 10 & $\mathrm{LT}$ & 0,032 & 0,084 & 0 \\
\hline 9 & 14 & LT & 0,013 & 0,270 & 0 \\
\hline 10 & 11 & LT & 0,082 & 0,192 & 0 \\
\hline 12 & 13 & LT & 0,221 & 0,200 & 0 \\
\hline 13 & 14 & $\mathrm{LT}$ & 0,171 & 0,348 & 0 \\
\hline 1 & 2 & $\mathrm{LT}$ & 0,019 & 0,059 & 0,053 \\
\hline 1 & 5 & LT & 0,054 & 0,223 & 0,049 \\
\hline 2 & 3 & $\mathrm{LT}$ & 0,047 & 0,198 & 0,044 \\
\hline 2 & 4 & $\mathrm{LT}$ & 0,058 & 0,176 & 0,037 \\
\hline 2 & 5 & $\mathrm{LT}$ & 0,057 & 0,174 & 0,034 \\
\hline 3 & 4 & $\mathrm{LT}$ & 0,067 & 0,171 & 0,035 \\
\hline 4 & 5 & $\mathrm{LT}$ & 0,013 & 0,042 & 0,013 \\
\hline
\end{tabular}


Tabela A.2 - Dados de nós (Tipo 1 - geração, tipo 2 - carga)

\begin{tabular}{|c|c|c|c|c|c|}
\hline \multirow{2}{*}{ Nó } & \multicolumn{2}{|c|}{ Tensão (pu) } & \multicolumn{2}{c|}{ Potência (MVA) } & \multirow{2}{*}{ Tipo } \\
\cline { 2 - 6 } & Módulo & Ângulo & Ativa & Reativa & \\
\hline 1 & 1 & 0 & 0 & 0 & 1 \\
\hline 2 & 1 & 0 & 0 & 0 & 1 \\
\hline 3 & & & 0 & 0 & 2 \\
\hline 4 & & & 17,79 & $-3,9$ & 2 \\
\hline 5 & & & 7,599 & 1,599 & 2 \\
\hline 6 & 1 & 0 & 0 & 0 & 1 \\
\hline 7 & & & 0 & 0 & 2 \\
\hline 8 & & & 0 & 6,9 & 2 \\
\hline 9 & & & 19,499 & 16,599 & 2 \\
\hline 10 & & & 9 & 5,799 & 2 \\
\hline 11 & & & 3,501 & 1,8 & 2 \\
\hline 12 & & & 6,099 & 1,599 & 2 \\
\hline 13 & & & 13,5 & 5,799 & 2 \\
\hline 14 & 1 & 0 & 14,901 & 5,001 & 3 \\
\hline
\end{tabular}

Tabela A.4 - Injeção Harmônica de corrente $\left(7^{a}\right.$ e $9^{a}$ harmônicas)

\begin{tabular}{|c|c|c|c|c|}
\hline \multirow{2}{*}{ Barra } & \multicolumn{2}{|c|}{$7^{a}$ harmônica } & \multicolumn{2}{c|}{$9^{a}$ harmônica } \\
\cline { 2 - 5 } & Módulo & $\begin{array}{c}\text { Ângulo } \\
(\mathrm{rad})\end{array}$ & Módulo & $\begin{array}{c}\text { Ângulo } \\
\text { (rad) }\end{array}$ \\
\hline 1 & 0 & 0 & 0 & 0 \\
\hline 2 & 0 & 0 & 0 & 0 \\
\hline 3 & 0,020 & 0,172 & 0,040 & 1,548 \\
\hline 4 & 0,040 & 0,071 & 0,080 & 0,639 \\
\hline 5 & 0,030 & 0,257 & 0,060 & 2,313 \\
\hline 6 & 0 & 0 & 0 & 0 \\
\hline 7 & 0,036 & 3,012 & 0,072 & 1,972 \\
\hline 8 & 0,040 & 0,081 & 0,080 & 0,729 \\
\hline 9 & 0,024 & 0,018 & 0,048 & 0,162 \\
\hline 10 & 0,026 & 0,140 & 0,052 & 1,260 \\
\hline 11 & 0,048 & 0,048 & 0,096 & 0,432 \\
\hline 12 & 0,020 & 0,069 & 0,040 & 0,621 \\
\hline 13 & 0,022 & 0,095 & 0,044 & 0,855 \\
\hline 14 & 0,028 & 0,089 & 0,056 & 0,801 \\
\hline
\end{tabular}

Tabela A.3 - Injeção Harmônica de corrente $\left(3^{a}\right.$ e $5^{a}$ harmônicas)

\begin{tabular}{|c|c|c|c|c|}
\hline \multirow{2}{*}{ Barra } & \multicolumn{2}{|c|}{$3^{a}$ harmônica } & \multicolumn{2}{|c|}{$5^{a}$ harmônica } \\
\hline & Módulo & $\begin{array}{l}\text { Ângulo } \\
\text { (rad) }\end{array}$ & Módulo & $\begin{array}{c}\text { Ângulo } \\
\text { (rad) }\end{array}$ \\
\hline 1 & 0 & 0 & 0 & 0 \\
\hline 2 & 0 & 0 & 0 & 0 \\
\hline 3 & 0,100 & 1,720 & 0,050 & 1,892 \\
\hline 4 & 0,200 & 0,710 & 0,100 & 0,781 \\
\hline 5 & 0,150 & 2,570 & 0,075 & 2,827 \\
\hline 6 & 0 & 0 & 0 & 0 \\
\hline 7 & 0,180 & & 0,090 & 1,712 \\
\hline 8 & 0,200 & 0,810 & 0,100 & 0,891 \\
\hline 9 & 0,120 & 0,180 & 0,060 & 0,198 \\
\hline 10 & 0,130 & 1,400 & 0,065 & 1,540 \\
\hline 11 & 0,240 & 0,480 & 0,120 & 0,528 \\
\hline 12 & 0,100 & 0,690 & 0,050 & 0,759 \\
\hline 13 & 0,110 & 0,950 & 0,055 & 1,045 \\
\hline 14 & 0,140 & 0,890 & 0,070 & 0,979 \\
\hline
\end{tabular}

Tabela A.5 - Injeção Harmônica de corrente $\left(11^{a}\right.$ e $13^{a}$ harmônicas)

\begin{tabular}{|c|c|c|c|c|}
\hline \multirow{2}{*}{ Barra } & \multicolumn{2}{|c|}{$11^{a}$ harmônica } & \multicolumn{2}{|c|}{$13^{a}$ harmônica } \\
\hline & Módulo & $\begin{array}{c}\text { Ângulo } \\
\text { (rad) }\end{array}$ & Módulo & $\begin{array}{c}\text { Ângulo } \\
\text { (rad) }\end{array}$ \\
\hline 1 & 0 & 0 & 0 & 0 \\
\hline 2 & 0 & 0 & 0 & 0 \\
\hline 3 & 0,010 & 1,376 & 0,010 & 0,516 \\
\hline 4 & 0,020 & 0,568 & 0,020 & 0,213 \\
\hline 5 & 0,015 & 2,056 & 0,015 & 0,771 \\
\hline 6 & 0 & 0 & 0 & 0 \\
\hline 7 & 0,018 & 2,102 & 0,018 & 2,752 \\
\hline 8 & 0,020 & 0,648 & 0,020 & 0,243 \\
\hline 9 & 0,012 & 0,144 & 0,012 & 0,054 \\
\hline 10 & 0,013 & 1,120 & 0,013 & 0,420 \\
\hline 11 & 0,024 & 0,384 & 0,024 & 0,144 \\
\hline 12 & 0,010 & 0,552 & 0,010 & 0,207 \\
\hline 13 & 0,011 & 0,760 & 0,011 & 0,285 \\
\hline 14 & 0,014 & 0,712 & 0,014 & 0,267 \\
\hline
\end{tabular}

